\title{
MAGNETIC RELAXATION IN HIGHLY ANISOTROPIC SYSTEMS*
}

\author{
A. J. Fedro \\ Materlals Sclence and Technology Dfvision \\ Argonne National Laboratozy, Argonne, Illinois 60439 \\ and \\ Northern Illinois University \\ DeKa1b, IL 60115 \\ and \\ B. D. Dunlap \\ Materials Sclence and Technology Division \\ Argonne National Laboratory, Argonne, Illiaois 60439
}

June 1995

CONF-850890--8

jing

$\mathrm{DE} 85 \quad 014992$

\section{DISCLAIMER}

\begin{abstract}
This report was prepared as an account of work sponsored by an agency of the United States Government. Neither the United States Government nor any agency thereof, nor any of their employees, makes any warranty, express or implied, or assumes any legal liability or responsibility for the accuracy, completeness, or usefulness of any information, apparatus, product, or process disclosed, or represents that its use would not infringe privately owned rights. Reference herein to any specific commercial product, process, or service by trade name, trademark, manufacturer, or otherwise does not necessarily constitute or imply its endorsement, recommendation, or favoring by the United States Government or any agency thereof. The views and opinions of authors expressed herein do not necessarily state or reflect those of the United States Government or any agency thereof.
\end{abstract}

Submitted to the International Conference on jnetism, sponsored by the International Union of Pure and Applied Physics, San Francisco, California, August 26-30, 1985.
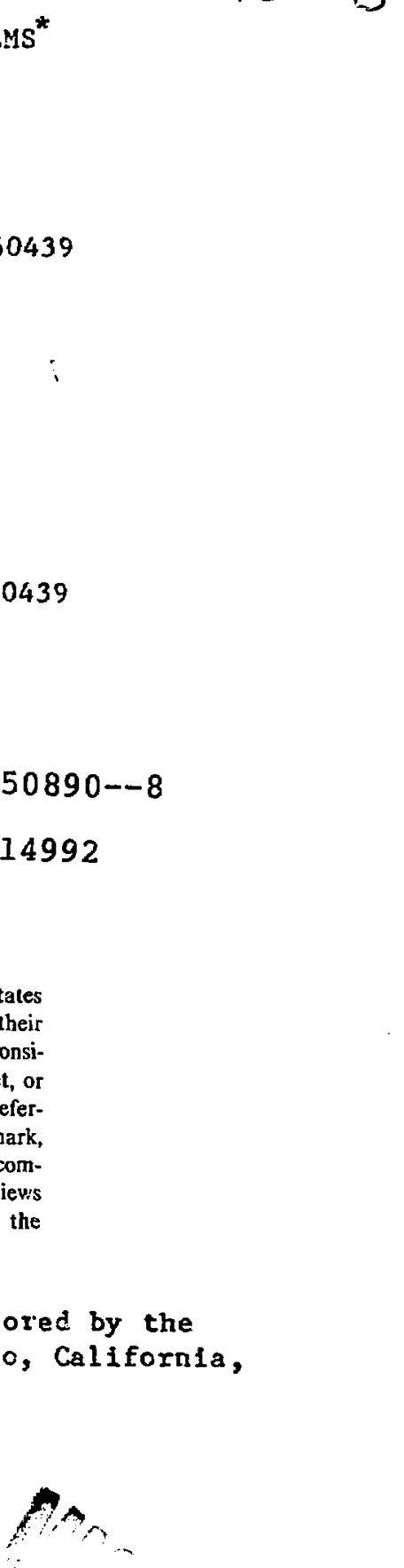

*Work supported by the U.S. Department of Energy.

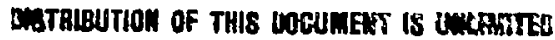

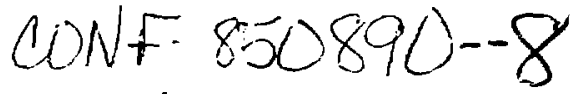


MAGNETIC RELAXATION IN HIGHLY ANISOTROPIC SYSTEMS

\author{
A. J. Fedro \\ Materials Sclence and Technology Division \\ Argonne National Laboratory, Argonne, Illinols 60439 \\ and \\ Northern Illino..s University \\ DeKalb, IL 60115 \\ and \\ B. D. Dunlap \\ Materials Sclence and Technology Division \\ Argonne Natlonal Laboratory, Argonne, Illinois 60439
}

\begin{abstract}
Using the Mori formalism, a general theory is developed for the calculation of paramagnetic relaxation effects in a general crystal field doublet. For systems with extreme magnetic anisotropy, the relaxation rates show a strong angular dependence, being severely peaked in a direction perpendicular to the magnetic easy axis. Application to the $R R h_{4} B_{4}(R=$ rare-earth) compounds is discussed.
\end{abstract}


While a very substantial body of 11 terature exists describing experimental results and theoretical interpretations for paranagnetic relaxation data, relatively little attention has been paid to situations where the system being investigated shows extreme magnetic anisotropy. Recent experimental work on $\mathrm{RRh}_{4} \mathrm{~B}_{4}$ ( $R=$ rare-earth) systems (1) have shown a number of anomalous features which available theoretical formulations are not able to describe. In the following we will discuss the general problem of spin relaxation in two-level systems, with application to highly anisotropic, Ising-like ground states. We consider a system of magnetic ions having angular momentum $J$ in a crystalline electric field (CEF) with a large axial anisotropy along a fixed (2) axis. If the sign of the CEF terms causes the large $J_{z}$ siates to be low-lying, and no offdiagonal CEF terms appear, then we generate a set of magnetic doublets where the ground state doublet is purely $J_{z}= \pm J$, the next doublet has $J_{z}= \pm(J-1)$ with energy separation $\Delta$, etc. If there are, in addition, small off-diagonal CEF terms and/or the system is in an applied field $H\left(\Delta \gg g_{J} \mu_{B} H\right)$ orfented at an angle $\theta$ wt th the $z$ axts, then each CEF doublet will have small admixtures of other 
angular momentum values. At temperatures small compared to the CEF splittings, this produces a system having very large magnetic anisotroples. It should be noted that relaxation cannot take place in the ground state doublet through normal $\Delta J_{z}= \pm 1$ processes without the admixture terms described above, if $\mathrm{J}>1$. Thus the nature of the ground state relaxation is different from that of simple $S=1 / 2$ doublets. We also note that mar.y such CEF situations are known in rare-earth compounds, although detailed spin relaxation data ere relativiciy 1infrequent.

In the following we consider temperatures small compared to the CEF splittings, $i . e$. we focus on the ground state doublet and consider an effective two level system. We begin with a complete set of angular momentum states $\{|m\rangle\}$ for each magnetic site defined by

$$
\mathrm{J}_{\mathrm{z}}|\mathrm{m}\rangle=\mathrm{m}|\mathrm{m}\rangle ; \quad(-\mathrm{J}<\mathrm{a}<\mathrm{J})
$$

and a Hamil tonian

$$
\mathcal{X}_{0}=y_{\mathrm{axIa1}}+\mathcal{X}^{\prime}-\mathrm{g}_{\mathrm{J}} \mu_{\mathrm{B}} \overrightarrow{\mathrm{H}} \cdot \overrightarrow{\mathrm{J}}
$$

The diagonal CEF operator $\mathcal{C}_{\text {axial }}$ is a function of $\mathrm{J}_{z}^{2}, \mathcal{X}$ ' contains the off-diagonal CEF terms Involving $J_{x}$ and $J_{y}$, and $H$ is the magnetic field applied at an arbitrary angle $\theta$ to the $z$ axis. The 
eigenstates of this Hamiltonian are written

$$
\mathcal{H}_{0}|M\rangle=E_{M}|M\rangle ; \quad|M\rangle=\sum_{m=-J}^{J} \lambda_{M m}|m\rangle .
$$

We consider the CEF parameters to be known.

Therefore, the solution of Eq. (2) gives the $E_{M}$ and $\lambda_{M m}$ as known functions of those CEF parameters, as well as of $H$ and $\theta$.

Transitions between the states |M which lead to relaxation are caused by some coupling to an external lattice, designated by $V$. We have considered the on-site exchange with the conduction electron system (Korringa mechanism), as well as a general dipolar-like coupling between the magnetic Ions which leads to concentration dependent effects in the relaxation. Both these interactions contain the operators $\vec{J}$ for the various sites, which we express at each site through the exact relation

$$
J_{\alpha}=\sum_{M, M^{\prime}}\left\langle M\left|J_{\alpha}\right| M^{\prime}\right\rangle|M\rangle\left\langle M^{\prime}\right| ; \alpha=x, y, z
$$

where the $\left\langle M\left|J_{\alpha}\right| M^{\prime}\right\rangle$ are easily obtained froal the known wave-fuctions $|M\rangle$. For our two level systen, we label the states as $|1\rangle$ and $|\overline{1}\rangle$, with $E_{-}>E_{1}$. Using Eqs. (3) and (4) and the completeness relation $|1\rangle\langle 1|+| \overline{1}\rangle\langle\bar{I}|=1$, we obtain 


$$
\begin{aligned}
\mathrm{J}_{\alpha}= & \frac{1}{2}\left[\left\langle 1\left|\mathrm{~J}_{\alpha}\right| 1\right\rangle+\left\langle\overline{1}\left|\mathrm{~J}_{\alpha}\right| \overline{1}\right\rangle\right]+\left\langle 1\left|\mathrm{~J}_{\alpha}\right| \overline{1}\right\rangle|1\rangle\left\langle\overline{1}\left|+\left\langle\overline{1}\left|\mathrm{~J}_{\alpha}\right| 1\right\rangle\right| \overline{1}\right\rangle\langle 1| \\
& +\frac{1}{2}\left[\left\langle 1\left|\mathrm{~J}_{\alpha}\right| 1\right\rangle-\left\langle\overline{1}\left|\mathrm{~J}_{\alpha}\right| \overline{\mathrm{l}}\right\rangle\right][|1\rangle\langle 1|-| \overline{\mathrm{l}}\rangle\langle\mathrm{T} 1] .
\end{aligned}
$$

From (5), it is clear that the ability of the lattice to induce transitions between the states $|1\rangle$ and $|\overline{1}\rangle$ depends on the coefficients $\left\langle 1\left|J_{\alpha}\right| \overline{1}\right\rangle=\left\langle\overline{1}\left|J_{\alpha}\right| 1\right\rangle$ which connect them.

The experimental systems of interest here are the compounds $Y_{1-c} R_{c} R_{4} B_{4}(c \ll 1 ; R=D y, H O)$. Using the CEF parameters of Dunlap et al., (2) we find $\left|\left\langle l\left|J_{x}\right| \bar{l}\right\rangle\right|,\left|\left\langle 1\left|J_{y}\right| \bar{I}\right\rangle\right|<10^{-5}$ for both Dy and Ho at all $\theta$ for $H<20$ koe. Such terms are therefore not effective in generating relaxation. For both Ho and Dy, we find $\left\langle 1\left|J_{2}\right| \overline{1}\right\rangle$ $<10^{-2}$ for $\theta \neq 90^{\circ}$. If, on the other hand, $\theta \cong 90^{\circ}$. we find for the non-Kramers ion (Ho), $\left\langle 1\left|J_{2}\right| \bar{I}\right\rangle \quad \sim 1$ in weak fields and shows a little field dependence while for the Kramers ion (Dy), $\left\langle 1\left|J_{z}\right| \bar{I}\right\rangle$ is small in weak fields, but is $\sim 1$ for only $H>10$ kOe. Physically, the peaking of relaxation around $90^{\circ}$. occurs from field-induced admixtures of other angular momentum states as the system moves from $J_{z}$ quantization to $J_{x}$ quantization.

From Eq. (5), we see that the angular momentum operators $J_{\alpha}$ are formed from the three basic excitations, $A_{V}$,

$$
A_{1}=\frac{1}{2}[|1\rangle\langle 1|-| \overline{1}\rangle\langle\bar{I} \mid\rangle], A_{2}=A_{3}{ }^{\dagger}=|1\rangle\langle\bar{I}| \text {. }
$$


Thus the evaluation of the response functions $\left(J_{\alpha}(t), J_{\alpha}(0)\right)$ requires the evaluation of $C_{\nu \nu^{\prime}}(t) \equiv\left(A_{v}(t), A_{\nu^{\prime}}(0)\right)$, where the notation $($,$) is that in$ the Mori formalism. (3) From standard Mori theory we find

$$
\frac{\partial}{\partial t} C_{\nu v^{\prime}}(t)+1 \omega_{v} C_{v \nu^{\prime}}(t)=-\sum_{v_{1}} \int_{0}^{t} d t^{\prime} \Gamma_{v v_{1}}\left(t-t^{\prime}\right) C_{v_{1} \nu^{\prime}}\left(t^{\prime}\right)
$$

where $w_{\nu}$ is the frequency associated with $A_{\nu}$ and the $\Gamma_{\nu v_{1}}(t)$ are the meinory functions associated with the interactions $V$ which cause the decay of $\mathrm{C}_{u v^{\prime}}(t)$. The Korringa mechanism leads to simple exponential decays of $\mathrm{C}_{\nu v^{\prime}}(\mathrm{t})$ with Lorentizian line shapes and no concentration dependence. The pair Interactions among the magnetic fons lead to a Gaussian $\left(t^{2}\right)$ time decay, dependent on the concentration.

To solve Eq. (7), we replace $C_{v v_{1}} v^{\prime}\left(t^{\prime}\right)$ by $c_{v_{1} v^{\prime}}(t)$. As pointed out by Abragam, (4) this gives accurate results for th absorption spectra (related to the Fourier transform of $\mathrm{C}_{\nu_{\nu^{\prime}}}$ ) in cases where the fluctuations in the local field as given by $\Gamma_{v v_{1}}(t)$ are either fast or slow. For slow fluctuations, the approximation is obvious. For fast fluctuations, the error lies far out in the wings of the absorption curve and of no interest. 
Within this approximation, we have

$$
c_{v v^{\prime}}(t)=e^{-i \omega} v_{v_{1}}\left[e^{-f(t)}\right]_{v v_{1}} c_{v_{1} v^{\prime}}(0) \text {, }
$$

with the decay being determined by the matrix

$$
\mathbf{f}_{v v_{1}}(t)=\int_{0}^{t} i t^{\prime} \exp \left[i\left(\omega_{\nu}-\omega_{v_{1}}\right) t^{\prime}\right] \int_{0}^{t^{\prime}} d t^{\prime \prime} \Gamma_{\nu v_{1}}\left(t^{\prime \prime}\right) \exp \left[i \omega_{v_{1}} t^{\prime \prime}\right]
$$

In the low frequency limit, we find

$$
f(t)=R_{K} t+G t^{2} / 2
$$

The first term is the Korringa contribution, with a relaxation matrix

$$
R_{K}=\frac{\pi}{2}\left[\frac{I_{\text {ex }} N(0)}{2}\right]^{2}\left(\frac{k_{B} T}{\hbar}\right) m_{K},
$$

where $m_{K}$ is a well-defined matrix involving matrix elements of the operators $J_{\alpha}$, and so is completely determined by the CEF.

The second term of Eq. (10) is related to pair Interactions among the magnetic lons, with $G$ depending on the nature of the interaction. For Heisenberg coupling, we find

$$
G=W \sum_{l_{1}}\left[\frac{{ }^{v_{\ell \ell}}}{h}\right]^{2}
$$

where $v_{\ell l_{1}}$ is the exchange coupling setween sites $\ell$ and $l_{1}$, and $W$ is again a well-defined matrix related to matrtx 
elements of $J_{\alpha^{*}}$ If $c \ll 1$, then a configurational average of this Gausian like behavior gives a simple exponential decay, as shown by McHenry et al. (5) That is,

$$
\left\langle\left\langle e^{-G t^{2} / 2}\right\rangle\right\rangle=e^{-R_{H} t}
$$

where

$$
\mathrm{R}_{\mathrm{H}}=c\left\langle\mathrm{v}^{2}\right\rangle^{1 / 2} \mathrm{~m}_{\mathrm{H}} ; \mathrm{m}_{\mathrm{H}}^{2}=\mathrm{N} \text {. }
$$

Thus $R_{H}$ is linear in the concentration, $c$, and proportional to the average exchange coupling. With Egs. (10) and (13), the configurationally averaged correlation functions become

$$
\left\langle\left\langle c_{v \nu^{\prime}},(t)\right\rangle\right\rangle=e^{-1 \omega} \nu^{t} \sum_{v_{1}}\left[e^{-\left(R_{K}+R_{H}\right) t}\right]_{\nu \nu_{1}} c_{v_{1} \nu^{\prime}}(0) .
$$

With this expression, the approprlate relaxation functions can be obtained for arbitrary crystal field interactions. We have outilned a general theory for the calculation of magnetic relaxation effects for a generalized CEF doublet, including both single fon and pair interaction mechanisms. Detalls of the calculation, as well as application to the $R R_{4} B_{4}$ data, will be published separately.

\section{ACKNOWLEDGMENTS}

We would like to acknowledge extensive discussions with F. Y. Fradin. This work was su: ported by the U.S. Department of Energy. 
References

1. K. Kumaga1, Y. Honda, and F. Y. Fradin, LT-17, U. Eckern, A. Schmid, W. Weber, and H. Wühl, eds., (North-Holland, 1984), P. 93; K. Kumagai, K. Kon, and F. Y. Fradin, 5 th International Conference on Crystaline Field and Anomalous Mixing Effects In f-electron Systems, Senda1, Japan, Apr11 $15-18,1985$.

2. B. D. Dunlap and D. Nlarchos, Solid State Commun. 44, 1577 (1982).

3. See for example, K. W. Becker, P. Fulde, and J. Keller, Z. Physik B 28, $9(1977)$.

4. A. Abragam, Principles of Nuclear Magnetisa, Clarendon Press, Oxford, 1962, Chapter X.

5. M. R. McHenry, B. G. Silbernagel, and J. H. Wernick, Phys. Rev. B5, 2958 (1972). 